\title{
Atomic and Ionic Radii of Superheavy Elements*
}

\author{
B. IRICKE AND J. T. WABER \\ Jepartment of Materials Science, Northwestern University, Livansion, Illinois 60201
}

(Received 30 August 1971)

\begin{abstract}
Atomic and ionic radii are presented for the elements E104-E120 and E156-E172. It is shown that a number of effects correlated with the large relativistic contraction of orbitals with low angular momentum leads to smaller atoms for higher atomic numbers. It is expected that $\mathrm{Cs}$ is the largest atom in nature.
\end{abstract}

A number of years ago, Waber and Cromer ${ }^{1}$ published a set of orbital radii of the known elements of the Periodic Table up to lawrencium. Subsequently ${ }^{2}$ element E104 has been isolated and evidence been presented $^{3,4}$ for E105.

The growing excitement about the possibility that elements on an island of nuclear stability $y^{5,6}$ around the atomic number E114, and those on maybe a second island ${ }^{6}$ around E164, might either exist or be possible to prepare, has lead us to calculate further radii for these superheavy elements.

The method Waber and Cromer ${ }^{1}$ used was to define the radius of the principal maximum of the outermost orbital of an atomic (or ionic) species as its atomic (or ionic) radius. The calculations itself were made by use of a self-consistent Dirac-Slater program described elsewhere. ${ }^{7,8}$ This set of radii was tested by Slater. ${ }^{9,10}$ In general, the agreement in approximately 1200 compounds was very good with experimental atomic radii which were deduced from interatomic distancesparticularly since no corrections for coordination number, electronegativity, etc., were made.

Table I contains the atomic and ionic radii we have deduced for the elements E104-E120, and to be complete we summarize in Table II the results for the elements of the second quasistable island E156-E172. The elements in between are expected to be extremely un- stable. Nevertheless, atomic radii for the first elements of this so-called superactinide series are already given. ${ }^{11}$ The probable principal valences and the electron configurations of the superheavy elements as well as their location in the Periodic Table were discussed in a recent paper. ${ }^{12}$

The cationic radii of the known elements are in good agreement with other estimates, so that the values presented in Tables I and II are expected to be good first approximations of the true values. It should be mentioned that these radii slightly depend on the value taken for the exchange approximation. Waber and Cromer $^{1}$ used the full value of Slater's exchange approximation which gives smaller values than the reduced exchange approximation we used. We chose the reduction factor $\frac{2}{3}$ as suggested by Kohn et al. ${ }^{13}$ which is especially true for very heavy elements as found by Rosén et $a l .{ }^{14}$ The difference in the calculated radii is normally very small (only a few percent) with the exemption of the alkaline and alkaline-earth elements; they will be discussed below.

The anionic radii tend to be too small by approximately $0.5 \AA$; that is, the cation and anion do not add to yield sufficiently large interatomic distances. That means that the radii of $\mathrm{E} 117^{-}$and $\mathrm{E} 171^{-}$are expected to be instead about 1.9 and $1.8 \AA$, respectively.

Two simple rules for the atomic and ionic radii in the 
TABLE I. Atomic and ionic radii (in Angstrom units) of the superheavy elements E104-E120.

\begin{tabular}{llllll}
\hline & Atomic & \multicolumn{3}{c}{ In } \\
Element & radius & \multicolumn{3}{c}{ Ionic state-ionic radius } \\
\hline E104 & 1.49 & $4^{+}$ & $0.70_{5}$ & & \\
E105 & 1.42 & $5^{+}$ & 0.68 & & \\
E106 & 1.36 & $6^{+}$ & $0.66_{5}$ & $4^{+}$ & 0.86 \\
E107 & 1.31 & $7^{+}$ & 0.65 & $5^{+}$ & 0.83 \\
E108 & 1.26 & $8^{+}$ & $0.63_{5}$ & $4^{+}$ & 0.80 \\
E109 & 1.22 & $6^{+}$ & 0.77 & $3^{+}$ & 0.83 \\
E110 & 1.18 & $6^{+}$ & 0.74 & $2^{+}$ & 0.80 \\
E111 & 1.14 & $1^{+}$ & 1.12 & $3^{+}$ & 0.76 \\
E112 & 1.10 & $1^{+}$ & 1.08 & $2^{+}$ & 0.75 \\
E113 & 1.31 & $1^{+}$ & 1.05 & & \\
E114 & 1.21 & $2^{+}$ & 1.00 & & \\
E115 & 1.77 & $1^{+}$ & 1.12 & & \\
E116 & 1.51 & $2^{+}$ & 1.04 & & \\
E117 & 1.38 & $3^{+}$ & 0.99 & $1^{-}$ & 1.41 \\
E118 & 1.31 & $4^{+}$ & 0.94 & & \\
E119 & 2.55 & $1^{+}$ & 1.23 & & \\
E120 & 2.16 & $2^{+}$ & 1.16 & & \\
\hline \hline
\end{tabular}

known part of the Periodic Table are the following: The radii become larger in the same chemical group for larger atomic numbers and the size becomes smaller within the same period during the filling of the same shell. In Figs. 1 and 2 the radii of the outer electrons of the seventh and eighth and ninth period are drawn, which shows that the second rule is still valid although the graph has changed drastically especially in Fig. 2 in comparison to the lower periods. Strong relativistic effects increase the contraction of the outer shells, which can be seen most of all for the large superactinide

TABLE. II. Atomic and ionic radii (in Angstrom units) of the superheavy elements E156-E172.

\begin{tabular}{|c|c|c|c|c|c|}
\hline \multirow{2}{*}{ Element } & \multirow{2}{*}{$\begin{array}{c}\text { Atomic } \\
\text { radius } \\
0.98\end{array}$} & \multicolumn{4}{|c|}{ Ionic state-ionic radius } \\
\hline & & $2^{+}$ & 0.84 & & \\
\hline E157 & 0.92 & $3^{+}$ & 0.81 & & \\
\hline E158 & 0.88 & $4^{+}$ & 0.78 & & \\
\hline E159 & 1.05 & $5^{+}$ & 0.76 & $1^{+}$ & 0.82 \\
\hline E160 & 1.00 & $6^{+}$ & 0.74 & $2^{+}$ & 0.80 \\
\hline E161 & 0.96 & $7^{+}$ & 0.71 & $3^{+}$ & 0.78 \\
\hline $\mathrm{E} 162$ & 0.93 & $4^{+}$ & 0.75 & & \\
\hline E163 & 0.89 & $5^{+}$ & 0.73 & & \\
\hline E164 & 0.86 & $4^{+}$ & 0.84 & & \\
\hline E165 & 2.08 & $1^{+}$ & 0.82 & & \\
\hline E166 & 1.78 & $2^{+}$ & 0.79 & & \\
\hline E167 & 1.59 & $3^{+}$ & 0.76 & & \\
\hline E168 & 1.48 & $4^{+}$ & 0.73 & & \\
\hline $\mathrm{E} 169$ & 1.39 & $5^{+}$ & 0.70 & & \\
\hline $\mathrm{E} 170$ & 1.33 & $6^{+}$ & 0.68 & & \\
\hline E171 & 1.28 & $1^{-}$ & 1.30 & & \\
\hline $\mathrm{E} 172$ & 1.24 & & & & \\
\hline
\end{tabular}

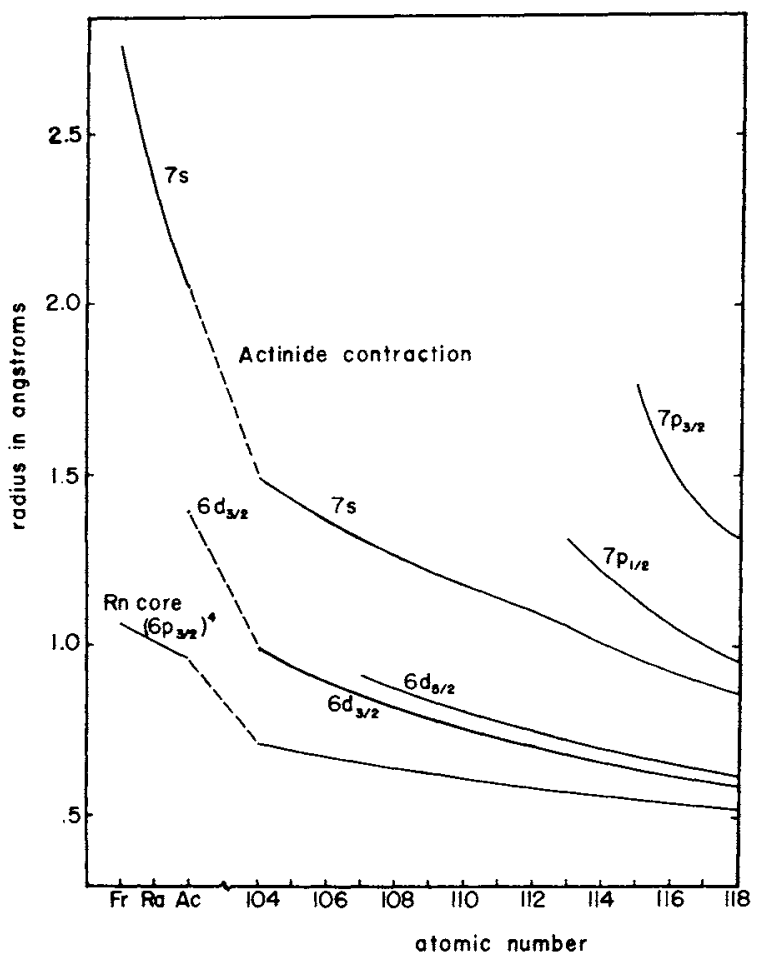

FIG. 1. The radii of the outer electrons of the fourth transition series, the elements Fr to E118.

contraction in Fig. 2. A second point is that the shells split into their two subshells with $j=l \pm \frac{1}{2}$ not only energetically but also spatially. The subshells with $j=l-\frac{1}{2}$ tend to be contracted more strongly whereas the subshells with $j=l+\frac{1}{2}$ are much less affected. As an

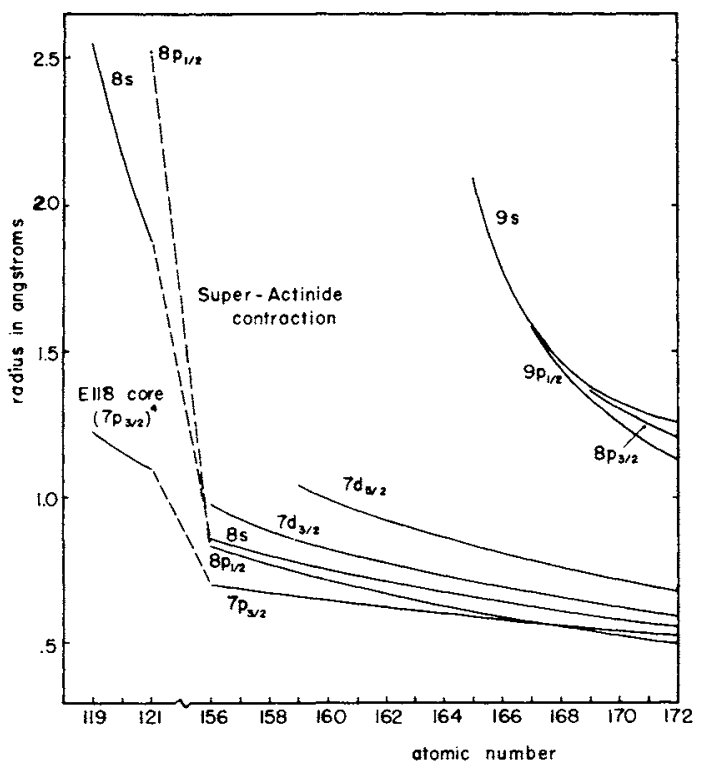

Fig. 2. The radii of the outer electrons of the eighth and ninth period, E119-E172. 


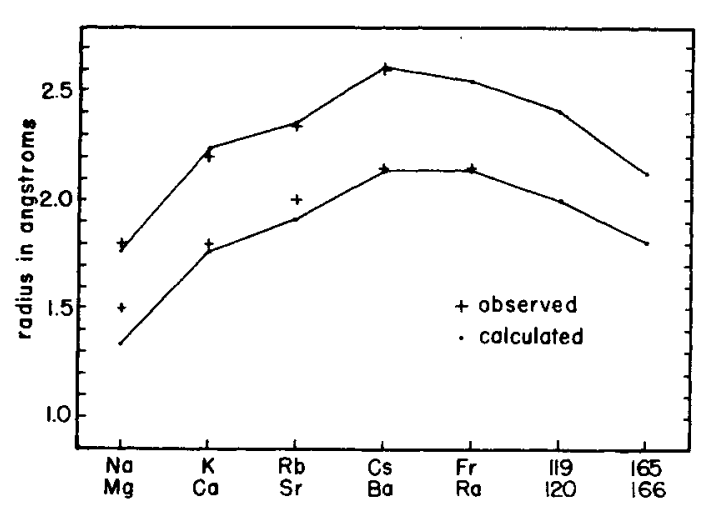

Fig. 3. Experimental and calculated atomic radii for the alkaline and alkaline-earth elements $\mathrm{Na}$ and $\mathrm{Mg}$ to E165 and E166. These values correspond to a value of 0.9 of Slater's exchange approximation to give the best agreement with the observed atomic radii.

extreme example of this separation, the $8 p_{1 / 2}$ shell starts to be filled at E121 and even lies below the $8 s$ shell for elements heavier than E126 whereas the $8 p_{3 / 2}$ subshell starts at E169 and has a radius which is larger by a factor of about 2.5 at these elements. The large contraction of the $8 s$ shell leads also to a very unusual sequence of the orbital radii of the outer electrons in the $7 d$ elements $\mathrm{E} 156-\mathrm{E} 164$ where the $7 d$ shell is the outermost shell.

The large decrease in radius for the high $s$ shells in the region of superheavy elements can be seen already at the radii of the alkaline and alkaline-earth elements which are presented in Fig. 3 and are compared with the experimental radii given by Slater. To yield the best agreement with the experimental values, we have chosen a factor of 0.9 for the exchange approximation because for these elements the radius changes by $15 \%$ for values of $\frac{2}{3}$ and 1 for Slater's exchange approximation. (However, to be consistent with our other calculations, we have drawn in Figs. 1 and 2 the radii for these elements calculated with the factor $\frac{2}{3}$.)

The first rule mentioned above that the atoms become larger in the same chemical group for higher atomic numbers is no longer valid. Already for $\mathrm{Fr}$ and $\mathrm{Ra}$ the increase of the radius stops and for E119 and E120, and even more so for E165 and E166, the radii decrease. This rule is also useless in the region of superheavy elements because the concept of a chemically analogous element in the same chemical group becomes more and more invalid, so that a comparison under this point of view is no longer possible. Because of the increasing number of shells available for bonding, the total separation of the normally known shells into their two subshells, the increasing relativistic effects even for the outer electrons, the increasing number of electrons with large angular momenta which can be filled deep inside the atom and the trend that many shells will be filled simultaneously, we expect that the radii of the atoms with very large hypothetic atomic numbers will asymptotically decrease and also the difference between different atoms will be much smaller than in the known part of the Periodic Table. This means that element Cs will be the largest atom which appears in nature.

Relative to Table I, Cunningham ${ }^{15}$ and also Keller and Burnett ${ }^{16}$ predicted the radius of the tetravalent form of E104 to be $0.75 \AA$ by extrapolating known experimental data. Keller and Burnet $t^{16}$ also predicted the radius of the monovalent cation of E113 to be $1.48 \AA$. Both these extrapolations give values in very good agreement with our calculated values.

* Work supported by a grant from the U.S. Atomic Energy Commission.

${ }^{1} \mathrm{~J}$. T. Waber and D. T. Cromer, J. Chem. Phys. 42, 4116 (1965).

${ }^{2}$ I. Zvara, Y. T. Chuburkov, R. Tsaletka, T. S. Tsarova, M. R. Shelevsky, and B. V. Shilov, Sov. J. At. Energy 21, 703 (1966).

${ }^{3}$ G. N. Flerov et al., JINR Preprint P7-3808, Dubna, 1968.

${ }^{4}$ A. Ghiorso et al., Phys. Rev. Letters 24, 1498 (1970).

${ }^{5}$ C. F. Tsang and S. G. Nielsson, Nucl. Phys. A140, 289 (1970).

${ }^{6} \mathrm{~J}$. Grumann, U. Mosel, B. Fink, and W. Greiner, Z. Physik 228, 371 (1969); J. Grumann, T. Morovic, and W. Greiner ibid. (to be published).

${ }_{7}$ D Liberman, J. T. Waber, and D. T. Cromer, Phys. Rev. 137, A27 (1965).

${ }^{8}$ D. Liberman, D. T. Cromer, and J. T. Waber, Computer Phys. Commun. 2, 107 (1971).

9 J. C. Slater, J. Chem. Phys. 41, 3199 (1964).

${ }_{10} \mathrm{~J}$. C. Slater, Quanium Theory of Molecules and Solids (McGraw-Hill, New York, 1965), Vol. 1, pp. $72 f f$.

${ }_{11}$ J. T. Waber, Proc. Robert A. Welch Foundation Conf. Chem. Res. 13th, Houston, 1969 (1970), pp. 353-443.

${ }_{12}$ B. Fricke, W. Greiner, and J. T. Waber, Theoret. Chim. Acta 21, 235 (1971); B. Fricke and J. T. Waber, Actinides Rev. 1, 433 (1971).

${ }^{13}$ W. Kohn and L. J. Sham, Phys. Rev. 140, A1113 (1965); R. Gàspàr, Acta Phys. Acad. Sci. Hung. 3, 263 (1964), 5, 65 (1955); R. D. Cowan, A. C. Larson, D. Liberman, J. A. Mann, and J. T. Waber, Phys. Rev. 144, 5 (1966).

${ }_{14}$ A. Rosén and I. Lindgren, Phys. Rev. 176, 114 (1968).

${ }_{15}$ B. B. Cunningham, see Ref. 140 of G. T. Seaborg, Ann. Rev. Nucl. Sci. 18, 53 (1968).

${ }^{16}$ O. L. Keller and J. L. Burnett, report by G. T. Seaborg on the Mendeleev Centennial in Ref. 11. 